\title{
Is there a Function for a Sex Pheromone Precursor?
}

\section{A Predicted Link between Bacterial Redox Metabolism and Propagation of Antibiotic Resistance}

\author{
${ }^{1}$ University of Liverpool, Crown street, Liverpool, UK, E-mail: ovasieva@ingenets.com \\ ${ }^{2}$ Ingenet Itd, 3d floor, 207 Regent street, London, UK, E-mail: ovasieva@ingenets.com \\ ${ }^{3}$ University of Edinburgh, Edinburgh, UK, E-mail: goryanin@oist.jp. https://orcid.org/0000-0002-8293-774X. \\ ${ }^{4}$ Okinawa Institute Science and Technology, Okinawa, Japan, E-mail: goryanin@oist.jp. \\ https://orcid.org/0000-0002-8293-774X. \\ 5 Tianjin Institute of Industrial Biotechnology, Tianjin, China, E-mail: goryanin@oist.jp. \\ https://orcid.org/0000-0002-8293-774X.
}

\begin{abstract}
:
Functional coupling and comparative genomics analysis have been applied to study functional associations of orthologs of enterococcal cAD1 sex pheromone (P13268) known to be responsible for biofilm formation, conjugative plasmid transfer and spreading of bacterial antibiotics resistance. CAD1 peptide pheromone is released from the membrane lipoprotein with the peptide precursor encoded by a gene cad ( $\operatorname{tr} \mid$ C2JQE7). Our analysis of genomic neighbourhood of cad and motifs of the encoded polypeptide and its orthologs suggests a close functional association between cAD1 and ApbE protein (Q82Z24), a FMN insertion and trafficking facilitator. The $c a d$ and $a p b E$ orthologs were coupled in the genomes and ApbE-specific motifs for FMN covalent attachment were identified in cad-encoded protein sequence and its orthologs. These findings suggest a potential role of FMN-based reductase function of the cAD1 lipoprotein precursor in its processing and release of the active sex pheromone peptide. They may lead to a new approach in prevention of antibiotic resistance spread via targeting sex pheromone processing chaperones or by suppression of the FMN availability and covalent binding. This methods can be also applied to a controlled evolution of bacterial pathogenicity in microbial fuel cells, as the findings suggest the crosstalk between bacterial pathogenicity and bacterial electro-activity.
\end{abstract}

Keywords: ApbE, cAD1, Enterococcus, redox metabolism, SEED

DOI: 10.1515/jib-2019-0016

Received: March 9, 2019; Revised: May 24, 2019; Accepted: June 7, 2019

\section{Introduction}

Recently developed insights in microbial redox metabolism [1], [2], [3] and emerged biotechnology of microbial fuel cells (MFC) [4] provided new data enabling to link cell redox processes and electron transfer to functions involved in virulence and bacterial pathogenicity, previously considered as largely autonomous. Suppression of pathogenic traits, observed in MFC under an applied electric potential [5], [6] in some groups of bacteria, is the fact that leaves more questions than answers. Functional links between bacterial redox metabolism and factors triggering biofilm formation and conjugational processes are coming to the focus of the research, and this in silico study establishes one of the potential connections.

Functional coupling of genes in bacterial genomes was demonstrated on multiple examples and is used to predict protein functions and new pathway connections [7], [8] A number of tool has been developed to support this analytical approach [7] and are at the moment among the most cited in the area of bacterial genomics. We have applied this method to infer functional connections of cAD1 sex pheromone (P13268) [9] produced by Enterococcus. As other known bacterial sex pheromones, cAD1 is secreted by plasmid-free cells to be recognised by plasmid-baring cells and initiate cell clumping, conjugation and the virulence pAD1 plasmid transfer [10], [11], [12]. This processes are tightly associated with biofilm formation and a number of other pathogenic determinants and are triggered by stressors, especially in a form of free radicals [13], [14], [15]. 
Conjugative transfer is the most efficient way of horizontal gene spread, and it is therefore considered one of the major reasons for the increase in the number of bacteria exhibiting multiple-antibiotic resistance [16]. Thus, conjugation and spread of antibiotic resistance represents a severe problem in antibiotic treatment, especially of immunosuppressed patients and in intensive care units. No consistent data exist on regulation of the pheromone peptide signalling by mechanisms associated with the metabolic state of a cell, its potential triggers and facilitators.

The known enterococcal sex pheromones (cAD1, cPD1, cCF10, cAM373, and cOB1) [15], [17] are all relatively hydrophobic, linear octa- or heptapeptides that are active at nanomolar concentrations. They all are proteolytically processed from the precursors-longer membrane-bound proteins with non- identified functions in different bacteria, as well as in fungi [18], [19]. Certain amino-acid motifs are conserved in pheromone lipoproteins within at least one orthology class [20].

\section{Workflow}

Our analysis of motifs of cAD1 sex pheromone precursor protein and its orthologs, as well as the genomic neighbourhoods of cad homologs in different genomes suggests close functional association between cAD1 and ApbE protein (Q82Z24), known to be involved in thiamine metabolism in Enterobacteria [21], recently associated with flavin trafficking in Treponema [22] and confirmed to be a FAD/FMN insertion chaperon [23], [24]. This suggests a potential role of FMN availability or its redox-dependent trafficking in the sex pheromone precursor processing and, consequently, biofilm formation, bacterial pathogenicity and antibiotic resistance.

A workflow for the presented analysis is depicted in Figure 1. SEED Viewer (PubSEED) and the associated comparative genomics platform (http://pubseed.theseed.org) have been used in this study. The SEED provides precise genome annotations that are cross-validated and unified across the genomes. It is a platform for a comparative functional and phylogenetic analysis of multiple sets of genomes and the basis for RAST annotation service. [7]. It also integrates information from validated sources (Kegg, NCBI, ProDom, etc) and manual curation, and can be explored in terms of sequences, pathways and functional subsystems analysis. The basic information and genomic regions containing homologs of cAD1 precursor encoding gene (UniProt: P13268) (SEED ID: fig|226185.9.peg.3033) were retrieved for the comparative analysis. 'Compare region' application has been used for the homologous bacterial genomic regions retrieval (set e-20 similarity threshold) and structural comparison. 'Compare regions' diagrams present homologous genes as same color-coded arrows with the same associated number (a direction of an arrow indicates the coding vector). The regions are aligned (pinned) via the seed gene homologs (presented as N1, red in all the diagrams).

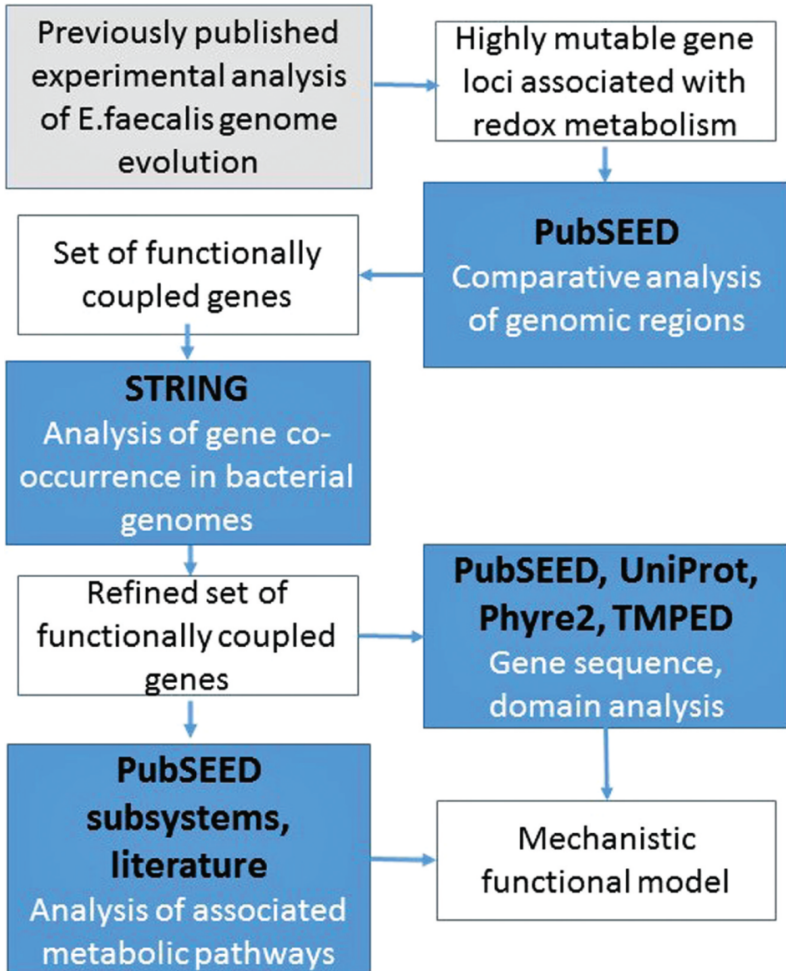


Figure 1: Illustration of the applied work flow.

SEED platform (http:/ / pubseed.theseed.org/) was used to retrieve and compare the bacterial genomic regions associated with genes of interest. Analysis of genes co-occurrence in the genomes and their connectivity were performed in STRING (https://string-db.org/) environment. SEED, STRING and Uniprot (https://www.uniprot.org/) tools were applied to analyse protein structural features and the relevant metabolic pathways.

STRING (http: / / string-db.org) analytical platform has been used to reconstruct functional associations between genes in the studied genomic regions by integration of known and predicted protein interactions [25]. The input proteins are presented graphically in the context of their interaction networks and phylogenetically associated patterns of the genomic neighbourhoods, gene co-occurrence and the expression profiles. Multiple protein names are used as an input and are mapped to the database organisms/genes via textual associations.

The input 'FAD:protein FMN transferase' and 'pheromone cad1' has been associated with Enterococcus faecalis among other organisms, and the following genes were chosen for the STRING analysis: EF_1225 - Thiamine biosynthesis ApbE; Flavin transferase that catalyzes the transfer of the FMN moiety of FAD and its covalent binding to the hydroxyl group of a threonine residue in a target flavoprotein (a.k.a. EF1225, NP_814952.1, 1200125, FAD:protein FMN transferase), EF_3256 - Pheromone cAD1 lipoprotein (a.k.a. NP_816853.1, EF3256, Q82Z23_ENTFA). The network was expanded by one more shell to automatically include the genes in the input genes genomic proximity: EF_3254-1,4-dihydroxy-2-naphtoate octoprenyltransferase and EF_3257 -Pyridine nucleotide-disulphide family oxidoreductase. These genes were selected in the reconstructed network and automatically added to the input list of functions.

'FAD:protein FMN transferase' and 'Electron transport complex rnf' input has been associated with Clostridium genus among other organisms, and the genes listed below were chosen for the following analysis: CD2483 - Hypothetical protein; Flavin transferase that catalyzes the transfer of the FMN moiety of FAD and its covalent binding to the hydroxyl group of a threonine residue in a target flavoprotein (a.k.a. CD630_24830, YP_001088997.1, AM180355, FAD:protein FMN transferase), rnfC - Electron transport complex protein (a.k.a. CD630_11370, YP_001087628.1, CD1137, Electron transport complex protein), rnfD - Electron transport complex protein (a.k.a. CD630_11380, YP_001087629.1, CD1138, Electron transport complex protein), rnfG - Electron transport complex protein (a.k.a. CD630_11390, YP_001087630.1, CD1139, Electron transport complex protein), rnfE - Electron transport complex protein (a.k.a. CD630_11400, YP_001087631.1, CD1140, Electron transport complex protein), rnfA - Electron transport complex protein (a.k.a. CD630_11410, YP_001087632.1, CD1141, Electron transport complex protein), rnfB - Electron transport complex protein (a.k.a. CD630_11420, CD1142, YP_001087633.1, Electron transport complex protein).

The 'neighbourhood' and 'co-occurrence' STRING views were chosen for graphical outputs of the associations between the input genes. For automated co-occurrence profiles STRING uses a phylogenetic profiling algorithm, SVD-Phy which performs truncated singular value decomposition to address the problem of uninformative profiles giving rise to false positive predictions. The graph shows all scores co-occurrences detected.

Phyre2 (Protein Homology/analogY Recognition Engine V 2.0 analysis, http://www.sbg.bio.ic.ac.uk/phyre2) was applied to reconstruct potential tertiary structure of cAD1. A crystal structure of cpe2226 protein from Clostridium perfringens, annotated in SEED as Putative pheromone precursor lipoprotein (fig |195102.1.peg.2289), and submitted by northeast structural genomics consortium (target cpr195) has been suggested by a search engine and used as a template for the 3D reconstruction with $99.9 \%$ confidence and $44 \%$ similarity to our query.

TMPRED (https://embnet.vital-it.ch/software/TMPRED_form.html) has been used for membranespanning motif prediction.

\section{Application}

\section{1 cAD1-ApbE Functional Coupling}

The Enterococcus faecalis gene locus containing cAD1 precursor (P13268) gene cad (tr|C2JQE7) represents an interesting combination of links to virulent and metabolic functions (Figure 2, Table 1). The two genes that are in an immediate proximity to cad encode for enzymes involved in electron transfer process or its modulation, and 3 other genes are relevant to quinone biosynthesis.

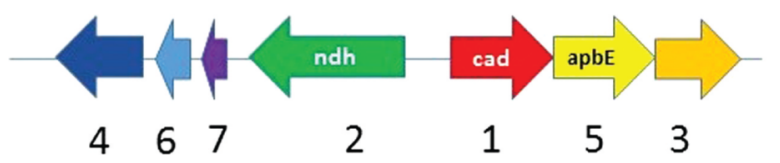


Figure 2: The Enterococcus faecalis gene locus containing cAD1 precursor (P13268) gene cad (tr |C2JQE7, fig | 226185.9.peg.3033). 7500 bp region is shown.

The arrows represent genes encoding for: 1- Pheromone cAD1 precursor lipoprotein Cad, 2- NADH dehydrogenase (EC 1.6.99.3), 3-1,4-dihydroxy-2-naphthoate polyprenyltransferase (EC 2.5.1.74),4- Heptaprenyl diphosphate synthase component I (EC 2.5.1.30), 5- Hypothetical similar to thiamine biosynthesis lipoprotein ApbE/FAD:protein FMN transferase (EC 2.7.1.180), 6- Heptaprenyl diphosphate synthase component I (EC 2.5.1.30), 7-hypothetical protein.

Table 1: Annotations and associated IDs for genes in cAD1 precursor/cad locus in Enterococcus faecalis V58 genome.

\begin{tabular}{|c|c|c|c|c|c|}
\hline$n$ & ID & Feature in SEED & bp & Function & FigFams \\
\hline 1 & $\operatorname{tr} \mid \mathrm{C} 2 \mathrm{JQE7}$ & fig | 226185.9.peg.3033 & 930 & $\begin{array}{l}\text { Pheromone cAD1 precursor } \\
\text { lipoprotein Cad }\end{array}$ & $\begin{array}{l}\text { FIG00132896: Pheromone cAD1 } \\
\text { precursor lipoprotein Cad }\end{array}$ \\
\hline 5 & $\operatorname{tr} \mid \mathrm{C} 0 \mathrm{X} 0 \mathrm{~V} 8$ & fig | 226185.9.peg.3032 & 1068 & $\begin{array}{l}\text { FAD:protein FMN transferase } \\
\text { (EC 2.7.1.180) @ FAD:protein } \\
\text { FMN transferase (EC 2.7.1.180), } \\
\text { HepST-associated }\end{array}$ & $\begin{array}{l}\text { FIG00132666: Hypothetical } \\
\text { similar to thiamin biosynthesis } \\
\text { lipoprotein ApbE }\end{array}$ \\
\hline 2 & $\operatorname{tr} \mid \mathrm{C} 0 \mathrm{X} 0 \mathrm{~V} 5$ & fig | 226185.9.peg.3034 & 1947 & $\begin{array}{l}\text { NADH dehydrogenase (EC } \\
\text { 1.6.99.3) in cluster with putative } \\
\text { pheromone precursor }\end{array}$ & $\begin{array}{l}\text { FIG01304395: NADH } \\
\text { dehydrogenase (EC 1.6.99.3) in } \\
\text { cluster with putative } \\
\text { pheromone precursor }\end{array}$ \\
\hline 3 & $\operatorname{tr} \mid$ C2JQE5 & fig | 226185.9.peg.3031 & 948 & $\begin{array}{l}\text { 1,4-dihydroxy-2-naphthoate } \\
\text { polyprenyltransferase (EC } \\
2.5 .1 .74)\end{array}$ & $\begin{array}{l}\text { FIG00085608: } \\
\text { 1,4-dihydroxy-2-naphthoate } \\
\text { polyprenyltransferase (EC } \\
2.5 .1 .74)\end{array}$ \\
\hline 6 & $\operatorname{tr} \mid \mathrm{COX0V3}$ & fig | 226185.9.peg.3036 & 558 & $\begin{array}{l}\text { Heptaprenyl diphosphate } \\
\text { synthase component I (EC } \\
\text { 2.5.1.30) }\end{array}$ & $\begin{array}{l}\text { FIG00004229: Heptaprenyl } \\
\text { diphosphate synthase } \\
\text { component I (EC 2.5.1.30) }\end{array}$ \\
\hline 4 & $\operatorname{tr} \mid \mathrm{Q} 82 \mathrm{Z19}$ & fig | 226185.9.peg.3037 & 987 & $\begin{array}{l}\text { Heptaprenyl diphosphate } \\
\text { synthase component II (EC } \\
\text { 2.5.1.30) }\end{array}$ & $\begin{array}{l}\text { FIG00002487: Heptaprenyl } \\
\text { diphosphate synthase } \\
\text { component II (EC 2.5.1.30) }\end{array}$ \\
\hline
\end{tabular}

'Feature in SEED' corresponds to an ID in SEED database, $n$ - number of a gene associated with Figure 1 genomic regions, aa-protein length, FigFams-protein family ID and its annotation in SEED database.

The genomic regions from different bacteria containing close homologs to cAD1 precursor (P13268) gene cad (Table 1, tr |C2JQE7) were retrieved from SEED database by means of 'Compare region' application with $\mathrm{e}^{-20}$ similarity thresholds set for region retrieval and color-coding of the homologs (Figure 3). The most striking information derived from this analysis was a strong positional association, with almost no exceptions between cad homologs and apbE homologs encoding thiamine biosynthetic protein ApbE/FAD:protein FMN transferase (Q82Z24) (Table 1, Figure 2-Figure 4). The conservative genomic regions (Table 1, Figure 2) also contained genes for: NADH-dehydrogenase 2 (N2, green arrows) as well as prenyltransferases and heptaprenyl diphosphate synthase subunits, representing elements of isoprenoid biosynthetic pathway (N 3, 4, 6-orange, blue, turquois arrows). The 1-6 module was conserved across the heterogeneous group of bacteria including Gram positive (diverse representatives of class Bacili (Lactobacillus, Listeria, Oenococus genera) and Clostridia, and Gram negative (classes of Clostridia (Catonella), Bacteroidetes (Bacteroides), (Spirochaete) representatives (Figure 3 and Figure 4). The core cluster was very conservative in all the Enterococci species (Figure 2 and Figure 5). However, 2 types of adjacent gene clusters were noticed (Figure 5). Only Enterococcus casseliflavus type, also including Enterococcus faecium, contain gene encoding for glutathione reductase $(\operatorname{tr} \mid \mathrm{COX} 0 \mathrm{U} 2)$ among the other differences in the immediate cluster proximity. 


\section{L. monocytogene \\ L.inocua \\ L.welshimeri ATCC \\ L.casei}

\section{E.faecium AusO \\ B.vulgatus EK4}

\section{C.morbi ATCC 5}

C.sp. 17-4

O.oeni PSU-1

T.oceani DSM 1

G.adiacens ATC

S.Ictaluri 707

\section{T.tengcongensi}

Ndh2 cAD1 ApbE

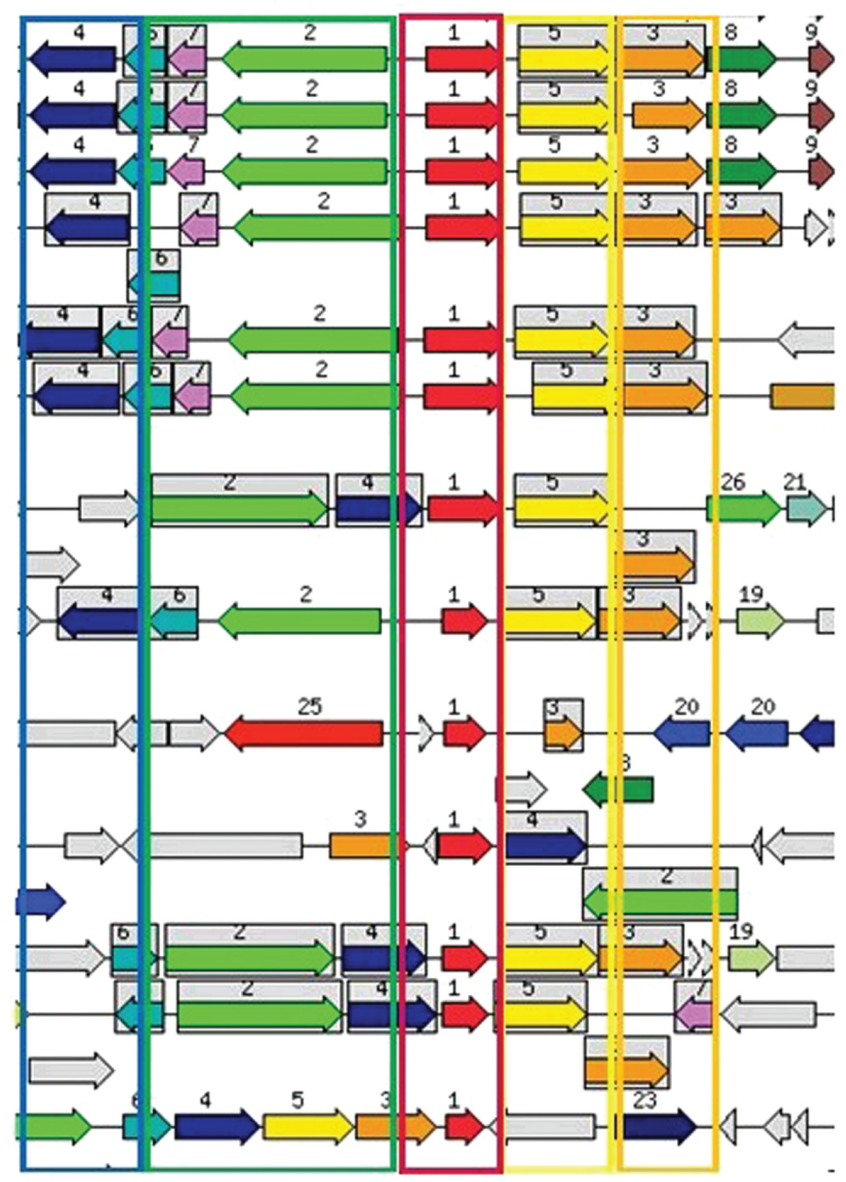

Figure 3: The genomic regions containing cAD1 precursor encoding cad gene and its close homologs retrieved from SEED database.

Seed gene: fig | 226185.9.peg.3033; e-20 similarity thresholds set for region retrieval and color-coding of the homologs. Regions shown correspond to 16,000 bp window. The arrows represent genes, same colour and number depicts homology, frames surround groups of homologous genes. Grey arrows represent genes that do not have homologs in the shown regions. The genes and encoded proteins are annotated in SEED as following: Red N1 - cad, Pheromone cAD1 precursor lipoprotein Cad; yellow N5-apbE, FAD:protein FMN transferase (EC 2.7.1.180); green, N2- NADH dehydrogenase (EC 1.6.99.3); orange, N3- 1,4-dihydroxy-2-naphtoate polyprenyltransferase (EC 2.5.1.74), N4- Heptaprenyl diphosphate synthase component II (EC 2.5.1.30). The other numbers define orthologous genes with no conserved genomic coupling to the cAD1 and functions not obviously related to the discussed metabolic area. Abbreviated species of bacteria from top to bottom (the left panel): Listeria (top 5 species), Lactobacillus casei, Enterococcus faecium, Bacteroides vulgatus, Catonella morbi, Oenococcus oeni, Thermosediminibacter oceani, Granulicatella adiacens, Streptococcus ictaluri, Thermoanaerobacter tengcongensis. 


\section{GENE NEIGHBORHOOD}

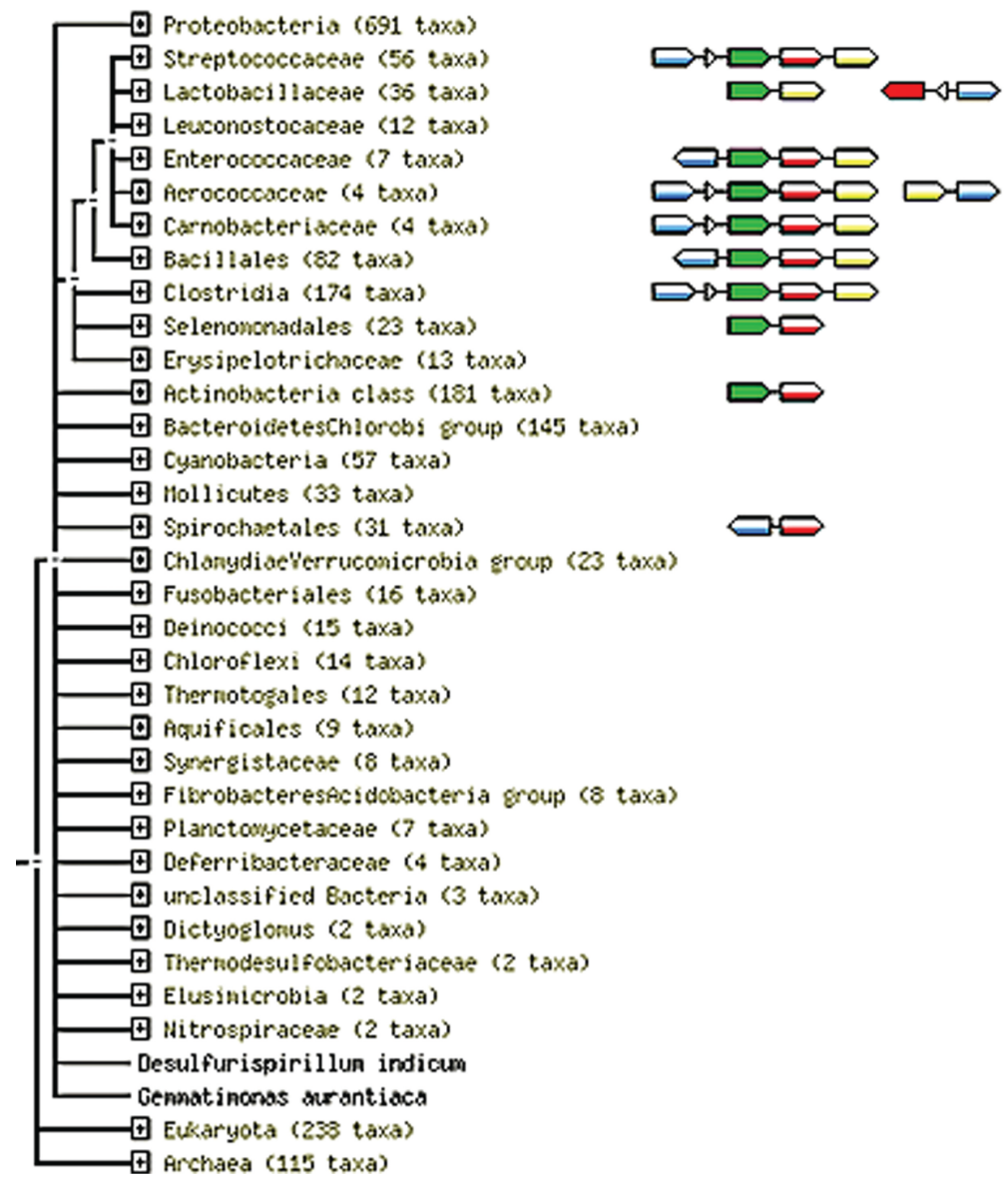

Figure 4: Co-occurrence and genomic proximity of genes encoding for homologs of cAD1 precursor (ref: Q82Z23, EF3256) and FAD: protein FMN transferase ApbE (ref: Q82Z24, EF1225).

String database, gene neighbourhood view. cAD1 orthologs are depicted as green arrows, 1,4-dihydroxy-2-naphtoate octoprenyltransferase (EF3254) -yellow arrows, and pyridine nucleotide-disulphide family oxidoreductase (EF3257)-blue arrows. The taxonomic tree used to display gene co-occurrence profiles was automatically generated by STRING and is based on canonical classification of all organism recorded in STRING database. The number of included taxa of low rank is shown in the brackets near each listed group name. STRING evaluates the phylogenetic distribution of orthologs of all proteins in a given organism. If two proteins show a high similarity in this distribution, i.e. if their orthologs tend to be observed as 'present' or 'absent' in the same subsets of organisms, then an association score is assigned [26].

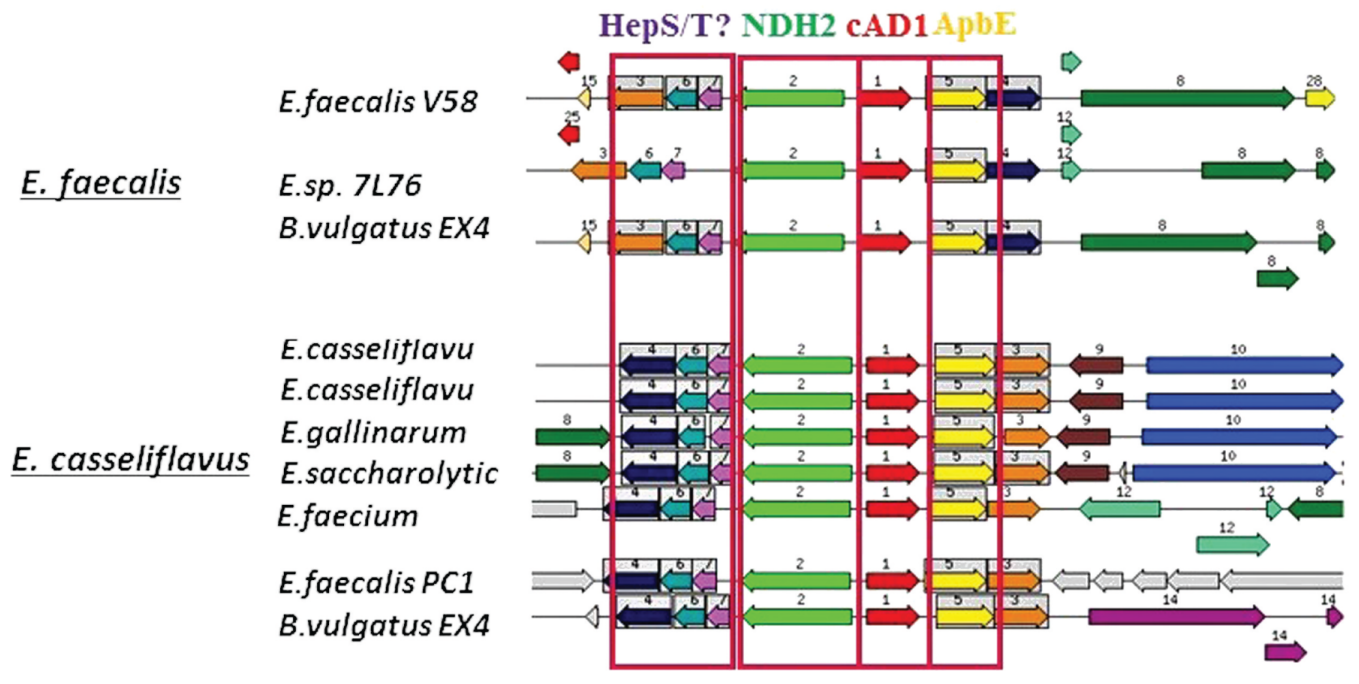


Figure 5: The genomic regions containing cAD1 precursor encoding cad genes from different Enterococci species (indicated at the beginning of each row) retrieved from SEED database.

The seed input gene: fig | 226185.9.peg.3033; e-20 similarity thresholds set for region retrieval and color-coding of the homologs. The arrows represent genes. Same frame and arrow colour and a number depict homologs. Regions shown correspond to $16,000 \mathrm{bp}$ window. Grey arrows represent genes that do not have homologs in the shown regions. Two types of the adjacent neighbourhoods are associated with the representative Enterococcus species (the left panel). Red N1 - cad, Pheromone cAD1 precursor lipoprotein Cad; yellow N5-apbE, FAD:protein FMN transferase (EC 2.7.1.180); green, N2NADH dehydrogenase (EC 1.6.99.3); purple, N7-HepS/T-heptaprenyl diphosphate synthase component, Red N1 - cad, Pheromone cAD1 precursor lipoprotein Cad; yellow N5-apbE, FAD:protein FMN transferase (EC 2.7.1.180); green, N2NADH dehydrogenase (EC 1.6.99.3); N4- Heptaprenyl diphosphate synthase component II (EC 2.5.1.30). The other numbers define orthologous genes with no conserved genomic coupling to the cAD1 and functions not obviously related to the discussed metabolic area.

\subsection{Coupling between ApbE and Redox Functions}

Analysis of functional coupling of $a p b E$ homologous genes via application of STRING [25] and SEED [7] have shown they consistently associated with $n q r(A-F)$ gene cluster in Gram negative bacteria possessing the encoded $\mathrm{Na}+$ transporting NADH-quinone oxidoreductase, such as Vibrionaceae (Vibrio, Shewanella, Ferrimonas) (Figure 6), Moraxellaceae (Psychrobacter). Interestingly, most of these genome have duplicated nqr clusters, and only one of them associated with apbE homolog.

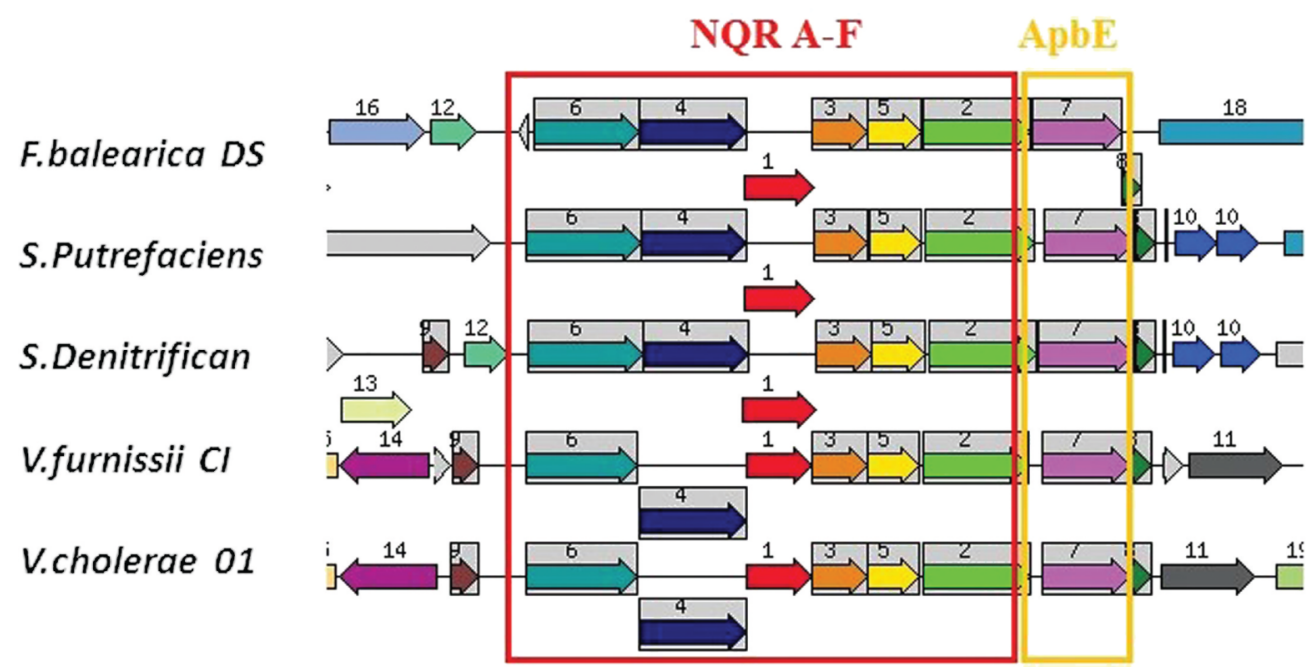

Figure 6: Example of genomic regions containing nqr A-F gene cluster and apbE orthologs retrieved from SEED database. Seed gene: nqrC, Ferrimonas balearica DSM (9799fig | 550540.3.peg.901); e-20 similarity thresholds set for region retrieval and color-coding of the homologs. The arrows represent genes, same arrow colour and a number depict homologs. Grey arrows represent genes that do not have homologs in the shown regions. Regions shown correspond to 16,000 bp window. The genes and encoded proteins are annotated in SEED as following: N1-6, framed,- Nqr A-F, Na(+)-translocating NADH-quinone reductase subunit (A-F) (EC 1.6.5.-), N7-apbE homologs- FAD:protein FMN transferase (EC 2.7.1.180) @ FAD:protein FMN transferase (EC 2.7.1.180), NqrBC-associated; Abbreviated species of bacteria from top to bottom (the left panel): Ferrimonas balearica DSM 9799, 2, 3-Shewanella species, 4,5-Vibrio species.

$A p b E$ homologs are also frequently associated with $r n f$ gene clusters, which is especially characteristic for Rnf-complex expressing Firmicutes, Actinobacteria, Bacteroidetes, Mollicutes and some representative of Fusobacteria (Sebaldella). A distant homology between cAD1 precursor and FMN-binding subunit of electron transfer Rnf complex, RnfG, has been mentioned [27], [28], [29] and suggests the evolutionary and/or functional link between RnfG and cAD1 precursor. In support of that, cad-apbE genomic associations appear only in genomes from which $r n f$ genes are missing (Figure 4).

Though nqr loci do not contain other genes typical for cad loci, rnf gene clusters are also coupled to 3 genes involved in isoprenoid biosynthesis (encoding for biosynthetic function in nonmevalonate branch of isoprenoid biosynthesis and-heptaprenyl-dipohosphat synthase) and some redox functions (as genes encoding for NAD/FAD utilising hydrogenase). Such similarity suggests some degree of functional redundancy between cAD1 precursor and Rnf complex, which is also based on a commonality of their dependence on covalently attached FAD/FMN cofactor. 


\subsection{FMN Binding Motifs in cAD1 Precursor}

FMN covalent-binding sites targeted by ApbE, such as in RnfG, share S(T)GAT amino acid motif, that is found to be required for ApbE chaperone activity [28]. They are different from the traditional non-covalent FMN binding sites existing in the majority of FMN-binding oxido-reductases [28]. We've also found single SGAT motifs in all annotated $\mathrm{NqrC}$ proteins, independently on their genomic coupling to ApbE homologs.

We used this motif to search for ApbE-lead FMN binding sites in cAD1 precursor. Two sites of FMN binding were found in two homologous domains of cAD1 precursor protein (Figure 7).

\section{MEWWGIKMKK KRFVTGFAVL AFSALVLGAC GADDNGSSNS SSESSTAQSS \\ TAKSTTESSA KVVAGGDLQD GTYKLEEKNY SNGYRAVFEM VVKDGKITES \\ KYDNVNENGE SKTKDAEYNK NMEAKSGTNP EKYIPELNDQ FLKAQSASGV \\ EVVTGATHSS ESFQNYAQQLIQAAQAGNTDTIEIDNGADL KDGTYKLEEK \\ NYSNGYRVQF EMTVAGGKVT ESNFDYIDKD GKSKQDDTEYNENMKAKSGT \\ EPKTYIPTLN DEFVKAMGEE DGSPANVEVV TGATHSSHSF IMYAQQLVNA \\ AEKGDTQTIE VDNIVTEK

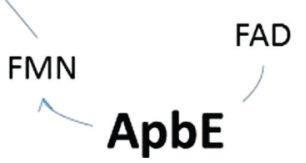

Figure 7: ApbE specific binding motifs for FMN insertion in cAD1 (P13268) sex pheromone protein precursor (SEED ID: fig | 226185.9.peg.3033).

The ApbE-dependent FMN binding sites are shown in bold, in red-the homologous sequences comprising the catalytic domain. The cAD1 peptide is shown in green. A signal sequence in shown in blue.

All the analysed protein sequences of cAD1 precursor orthologs from other bacteria had one or two SGAT motifs. For instance, two SGAT motifs are found in ortholohous Listerial pheromone protein precursors (ref (SEED): fig|386043.6.peg.2514 Pheromone cAD1 precursor lipoprotein Cad (Listeria welshimeri serovar $6 b$ str. SLCC5334)), Lactobacillus (ref (SEED): fig |525337.3.peg.1495 Pheromone cAD1 precursor lipoprotein Cad (Lactobacillus paracasei subsp. paracasei ATCC 25302)) and one SGAT motif is detected in Oenococcal ortholog (ref (SEED): fig | 203123.5.peg.1084 Pheromone cAD1 precursor lipoprotein Cad Oenococcus oeni PSU-1).

Similar, TGAS and TGAV motifs are identified in succinate dehydrogenase subunits, where the first motif is also associated with covalent FAD/FMN binding [24]. ApbE may potentially have a central function in metabolism of the discussed bacteria enabling specific covalent flavin-based electron transfer and, potentially, flavin trafficking.

Phyre2 (Protein Homology / analogY Recognition Engine V 2.0 analysis) has been applied to reconstruct potential tertiary structure of cAD1. A crystal structure of cpe2226 protein from Clostridium perfringens, annotated in SEED as Putative pheromone precursor lipoprotein (fig|195102.1.peg.2289), and submitted by northeast structural genomics consortium (target cpr195) has been suggested by a search engine and used as a template for the 3D reconstruction with $99.9 \%$ confidence and $44 \%$ similarity to our query. Interestingly, the closest functionally annotated match (98\% confidence and 17\% similarity with cAD1 within 56-163 residues) was Na+translocating NADH-quinone reductase subunit C (nqrc) from Shewanella and Vibrio Cholera (Figure 8).
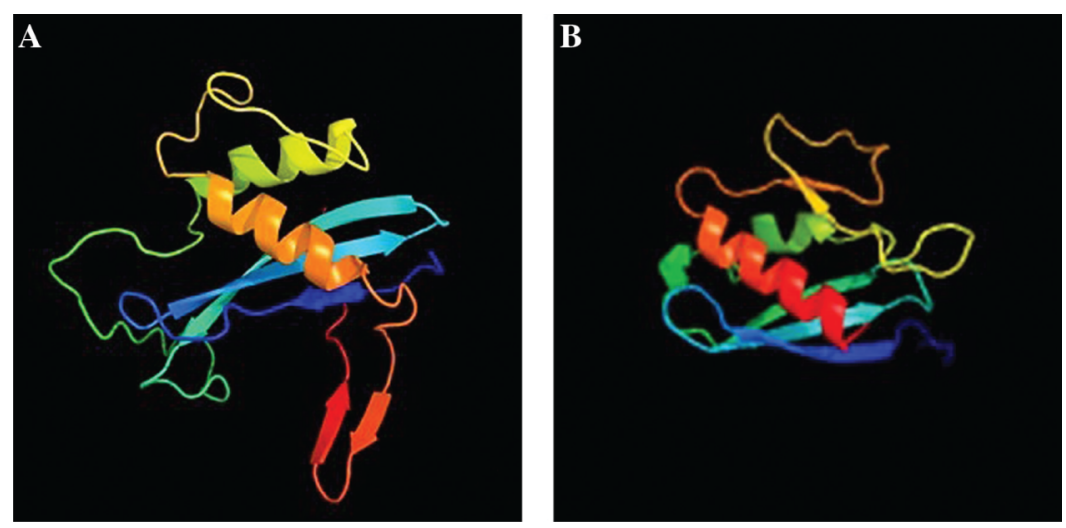
Figure 8: Results of comparison of the reconstructed tertiary structure of cAD1 to the library of known 3D protein structures via Phyre2 (Protein Homology/analogY Recognition Engine V 2.0 analysis).

A crystal structure of cpe2226 protein from Clostridium perfringens, cAD1 homolog annotated in SEED as Putative pheromone precursor lipoprotein (fig | 195102.1.peg.2289) was used as a template for the 3D reconstruction (44\% similarity to the query). 117 residues ( $38 \%$ of the sequence) have been modelled with $99.9 \%$ confidence by the single highest scoring template. A-reconstructed tertiary structure of cAD1, Model dimensions (Å):X:33.109 Y:47.972 Z:39.437; B-3D reconstruction of NqrC subunit of $\mathrm{Na}+$ translocating $\mathrm{NADH}$-quinone reductase based on crystal structure of Shewanella oneidensis $\mathrm{NqrC}$. Models are colored by rainbow from $\mathrm{N}$ to $\mathrm{C}$ terminus.

Tertiary structure of cAD1 125-291 residue part was also found to have 11\% similarity to Na+-translocating Nadh-quinone reductase 2 subunit C from Parabacteroides distasonis (yp_001302508.1). cAD1 possesses one transmembrane motif between 6-20 amino acids, identified by TMPRED program in cAD1 with a significant score of 1573.

Detailed and integrative structural analysis of the full polypeptide will be required for more meaningful functional prediction, however, as seen from the Figure 8 cAD1 and Na+ translocating NADH-quinone reductase subunit $\mathrm{C}$ share certain structural features.

\section{Discussion}

Unlike the majority of oxidoreductases that contain non-covalently associated FMN molecule, some redoxrelated enzymes contain covalently-bound cofactor, which receives electrons from NADH, and a tightly bound ubiquinone that mediates electron transfer from FAD to the diffusible quinone pool [30]. It has been recently shown that the ApbE thiamine biosynthetic protein homolog [21] is required for an attachment of an FMN residue to proteins via a phosphoester bond [23], [24], [31] and is also involved in extracellular flavin trafficking [22]. RnfG and NqrC subunits of the redox complexes, examples of covalently flavinated proteins [22], [24], [29], possess an ApbE-specific FMN insertion motif (TGAT) [24], [28] and are functionally coupled to ApbE homologs (Figure 6).

Enterococcal sex-pheromone lipoprotein precursor, cAD1, also occurred to be a FAD/FMN-binding protein. According to our analysis the cAD1 precursor and its orthologs from other bacteria are encoded by genes adjacent to $a p b E$ homologs (Figure 2-Figure 5). A number of mentioned above Firmicutes genera [32], [33] and particularly Listeria [34], Catonella [35] representatives, as well as Bacteroidia (Bacteroides) [36], were considered as electrogenic and shown to populate MFCs [4] under different conditions. We show that cAD1 precursor has two sites specific for ApbE-dependent FMN insertion (TGAT) (Figure 7), and its homologs have at least one FMN covalent binding insertion motif. Structurally, cAD1 shares features typical for NqrC subunit of $\mathrm{Na}+$ translocating NADH-quinone reductase (Figure 8) and possesses one transmembrane motif. In electroactive bacteria these proteins may comprise a part of a flavin trafficking [22], [37], external electron transfer cycle (Figure 9) and, hypothetically, serve a signalling function. Availability of extracellular flavins, as a measure of a communal density and of it's integral metabolic potential, may be translated into cAD1 precursor activity and has effect on its accessibility for proteases.

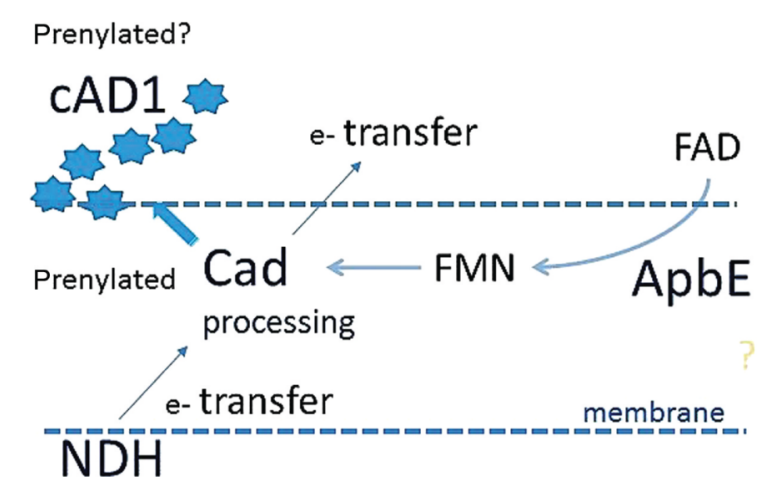

Figure 9: Suggested functional interaction between cAD1 pheromone and ApbE/FAD:protein FMN transferase. The release of cAD1 peptide pheromone may depend on a FMN-based electron transfer activity or a conformation of the lipoprotein precursor (Cad). ApbE works as a chaperone facilitating covalent binding of FMN and FAD/FMN extracellular trafficking.

A number of studies demonstrated an importance of flavin trafficking for electrogenic metabolism in bacteria [38]. From our analysis and the information discussed above, it would be logical to suggest that the particular 
sex pheromone systems can be also directly linked to the redox regulation and bacterial electroactivity. Interestingly, many bacteria that possess Nqr or Rnf complexes or cAD1 homologs are known as electrogens. Presence of these clusters and, particular, $c a d-a p b E$ gene cluster in a genome may be used as a predictive measure. There are still no reports for electroactivity of Oenococcus genera but, based our analysis, for instance, we can suggest that this genus also has electrogenic properties. We may even suggest, taking in mind pheromone properties of cAD1, that its processing can be one of regulators of relevant bacteria response to an applied electric potential and, for these bacteria groups, a trigger of the anodic biofilm formation in MFC [37], [39].

The analysis of the cad gene functional coupling suggests that prenylation of the terminal cysteine [40] is also involved in the processing of cAD1 peptide and in the electron transfer function of the pheromone precursor [30], which can be taken in account in development of new drugs supressing propagation of antibiotic resistance, for instance, based on inhibitors of isoprenoid biosynthesis. Nqr genes, typical for rather Gram negative bacteria possessing periplasm, were not found to be functionally coupled to metabolism of isoprenoids. It suggests that potential prenylation function associated with Rnf or cAD1 orthologs may reflect a need in membrane anchoring of these or other components of the protein complexes on the membrane.

Ironically, while we were preparing our manuscript, the publication in Nature appeared [41] that experimentally proved a link of Listeria pheromone PlpA (homologous to cAD1 according to our analysis) to the electron transfer and favin trafficking. This striking coincident indicates the timely significance of the described phenomenon, and we see two studies as pretty much complementary. Our enquiry has been commenced after the earlier research [42] where analysis of an advantage of Enterococcus faecalis over Staphylococcus aureus in a mixed host residential population pointed to an importance of the cad locus. In this work [42], NADH dehydrogenase gene $(\operatorname{tr} \mid \mathrm{COX} 0 \mathrm{~V} 5, \mathrm{~N} 2$ in Table 1$)$ was shown to be among the most frequently mutated in E. faecalis genome under the mixed population experimental conditions. These data support the involvement of cAD1 precursor locus in overall bacterial pathogenicity, competition and resistance.

A relation of the suggested electron transfer function of cAD1 precursor to spreading of antibiotic resistance in natural bacterial populations would be of a medical and biotechnological importance. It is especially intriguing, due to an extreme virulence of bacteria baring this sex pheromone system (among which are Enterococcus, Streptococcus, Staphylococcus) and documented spreading of antibiotic resistance in-between these species [43]. Similar to cAD1's ones, active or even cryptic motifs may be also discovered in evolutionary related proteins which can constitute new drug targets in other pathogens.

Antibiotics to which the conjugative transfer in Enterococcus is especially sensitive, such as vancomycin [44] are macrolides whose antibacterial action is based on cell membrane potential disturbance [3], which supports a suggested relevance of sex pheromone release to redox sensing. We propose that FMN/FAD binding by a specific motif in a particular sex pheromone lipoprotein precursor may regulate its processing and a consequent release of the peptides. We claim that $\mathrm{ApbE}$ and the associated cofactors may be important regulators of conjugative transfer and biofilm formation in Enterococcus, Staphylococcus, Streptococcus and Listeria and can be explored for new approaches to stop propagation of antibiotic resistance in chronic diseases [1], [2], [3], [10], [11], [12], [13], [14], [16], [17], [18], [41], [42], [43], [45]. The method applied here also demonstrates a potential of Insilco prediction of new functions and functional links for well-known proteins. It was shown to be effective in application to missing links in biochemical pathways [7], [8], However, genomic functional coupling paradigm may also change our understanding of the landscape of biological functional interactions on a larger scale opening new approaches for modulation of cellular and organismal characteristics.

\section{Funding}

Okinawa Institute of Science and Technology Graduate University, Funder Id: http:/ /dx.doi.org/10.13039/501100004199, Grant Number: BSU.

Conflict of interest statement: Authors state no conflict of interest. All authors have read the journal's publication ethics and publication malpractice statement available at the journal's website and hereby confirm that they comply with all its parts applicable to the present scientific work.

Authors' contributions: OV commenced the study and performed comparative genomics analysis, both authors have analysed the data and drafted the manuscript. 


\section{References}

[1] Sporer A], Kahl L], Price-Whelan A, Dietrich LEP. Redox-based regulation of bacterial development and behavior. Annu Rev Biochem 2017;86:777-97.

[2] Reniere ML. Reduce, induce, thrive: bacterial redox sensing during pathogenesis. ] Bacter 2018;200:e00128-18.

[3] Reese AT, Cho EH, Klitzman B, Nichols SP, Wisniewski NA, Villa MM, et al. Antibiotic-induced changes in the microbiota disrupt redox dynamics in the gut. eLife 2018;7:e35987.

[4] Kracke F, Bin L, Shiqin L, Jens Y, Krömer JO. Balancing cellular redox metabolism in microbial electrosynthesis and electro fermentation a chance for metabolic engineering. Metab Eng 2017;45:109-20.

[5] Yazdi H, Alzate-Gaviria L, Ren Z]. Pluggable microbial fuel cell stacks for septic wastewater treatment and electricity production. Bioresour Technol 2015;180:258-63.

[6] leropoulos I, Pasternak G, Greenman ]. Urine disinfection and in situ pathogen killing using a Microbial Fuel Cell cascade system. PLoS One 2017;12:e0176475.

[7] Overbeek R, Olson R, Pusch CD, Olsen C], Davis ]], Disz T, et al. The SEED and the Rapid Annotation of microbial genomes using Subsystems Technology (RAST). Nucl Acid Res 2014;42:D206-14.

[8] Melnick ], Lis E, Park ]H, Kinsland C, Mori H, Baba T, et al. Identification of the two missing bacterial genes involved in thiamine salvage: thiamine pyrophosphokinase and thiamine kinase. ] Bacteriol 2004;186:3660-2.

[9] An FY, Clewell DB. Identification of the CAD1 sex pheromone precursor in Enterococcus faecalis.] Bacteriol 2002;184:1880-7.

[10] Dunny GM, Brown BL, Clewell DB. Induced cell aggregation and mating in Streptococcus faecalis: evidence for a bacterial sex pheromone. Proc Natl Acad Sci USA 1978;75:3479-83.

[11] Dunny CM, Craig RA, Carron R, Clewel LDB. Plasmid transfer in Streptococcus faecalis: production of multiple sex pheromones by recipients. Plasmid 1979;2:454-65.

[12] Dunny CM, Funk C, Adsit ]. Direct stimulation of the transfer of antibiotic resistance by sex pheromones in Streptococcus faecalis. Plasmid 1981;6:270-8.

[13] Wirth R. The sex-pheromone system of Enterococcus faecalis - more than just a plasmid-collection mechanism. Eur] Biochem 1994:222:235-46.

[14] Dunny GM. Enterococcal sex pheromones: signaling, social behavior, and evolution. Annu Rev Genet 2013;47:457-82.

[15] Clewell DB, Weaver KE, Dunny CM, Coque TM, Francia MV, Hayes F. Extrachromosomal and mobile elements in enterococci: transmission, maintenance, and epidemiology. In: Gilmore MS, Clewell DB, Ike Y, Shankar N, editors. Enterococci: from commensals to leading causes of drug resistant infection. Boston, MA: Massachusetts Eye and Ear Infirmary, 2014.

[16] Grohmann E, Muth G, Espinosa M. Conjugative plasmid transfer in gram-positive bacteria. Microbiol Mol Biol Rev 2003;67:277-301.

[17] Clewell DB, Dunny GM. Conjugation and genetic exchange in enterococci. In: Cilmore MS, Courvalin PD, Clewell B, Dunny GM, Murray BE, Rice LB, editors. The enterococci: pathogenesis, molecular biology and antibiotic resistance, Washington, D.C.: ASM Press, 2002. 432p.

[18] Cook LC, Federle M]. Peptide pheromone signaling in Streptococcus and Enterococcus. FEMS Microbiol Rev 2014;38:473-92.

[19] Jones SK, Bennett R]. Fungal mating pheromones: choreographing the dating game. FC \& B 2011;48:668-76.

[20] Clewell DB, An FY, Flannagan SF, Antiporta M, Dunny GM. Enterococcal sex pheromone precursors are part of signal sequences for surface lipoproteins. Mol Microbiol 2000;35:246-7.

[21] Beck B], Downs DM. A periplasmic location is essential for the role of the ApbE lipoprotein in thiamine synthesis in Salmonella typhimurium. J Bacteriol. 1999;181:7285-90.

[22] Deka RK, Brautigam CA, Liu WZ, Tomchick DR, Norgard MV. Molecular insights into the enzymatic diversity of flavin-trafficking protein (Ftp; formerly ApbE) in flavoprotein biogenesis in the bacterial periplasm. Microbiologyopen. 2016;5:21-38.

[23] Fang X, Liang P, Raba DA, Rosas-Lemus M, Chakravarthy S, Tuz K, et al. Kinetic characterization of Vibrio cholerae ApbE: Substrate specificity and regulatory mechanisms. PLoS One 2017;12:e0186805.

[24] Bertsova YV, Fadeeva MS, Kostyrko VA, Serebryakova MV, Baykov AA, Bogachev AV. Alternative pyrimidine biosynthesis protein ApbE is a flavin transferase catalyzing covalent attachment of FMN to a threonine residue in bacterial flavoproteins. J Biol Chem 2013;288:1427686.

[25] Szklarczyk D, Franceschini A, Kuhn M, Simonovic M, Roth A, Minguez P, et al. The STRING database in 2011: functional interaction networks of proteins, globally integrated and scored. Nucl Acid Res 2011;39:D561-8.

[26] Szklarczyk D, Morris ]H, Cook H, Kuhn M, Wyder S, Simonovic M, et al. The STRING database in 2017: quality-controlled protein-protein association networks, made broadly accessible. Nucleic Acids Res 2017;45(Database issue):D362-8.

[27] Beck B], Downs DM. The apbE gene encodes a lipoprotein involved in thiamine synthesis in salmonella typhimurium. ] Bacteriol 1998;180:885-91.

[28] Backie ], Juárez O, Zagorevski DV, Wang Z, Nilges M], Barquera B. Covalent binding of flavins to RnfC and RnfD in the Rnf complex from Vibrio cholera. Biochem 2008;47:11273-84.

[29] Steuber ], Halang P, Vorburger T, Steffen W, Vohl FC. Central role of the Na+-translocating NADH:quinone oxidoreductase (Na+-NQR) in sodium bioenergetics of Vibrio cholera. Biol Chem 2014;395:1389.

[30] Messner KR, Imlay JA. the identification of primary sites of superoxide and hydrogen peroxide formation in the aerobic respiratory chain and sulfite reductase complex of Escherichia coli. ] Biol Chem 1999;274:10119-28.

[31] Boyd JM, Endrizzi JA, Hamilton TL, Christopherson MR, Mulder DW, Downs DM, et al. FAD binding by ApbE protein from Salmonella enterica: a new class of FAD-binding proteins. J Bacteriol 2011;193:887-95.

[32] Ng KK, Shi X, Ong SL, Ng HY. Pyrosequencing reveals microbial community profile in anaerobic bio-entrapped membrane reactor for pharmaceutical wastewater treatment. Bioresour Technol 2016;200:1076-9. 
[33] Kiseleva L, Garushyants SK, Ma H, Simpson D], Fedorovich V, Cohen MV, et al. Taxonomic and functional metagenomic analysis of anodic communities in two pilot-scale microbial fuel cells treating different industrial wastewaters. J Integr Bioinform 2015;12:275.

[34] Pierra M, Golozar M, Zhang X, Prévoteau A, De Volder M, Reynaerts D, et al. Crowth and current production of mixed culture anodic biofilms remain unaffected by sub-microscale surface roughness. Bioelectrochem 2018;122:213-20.

[35] Toczyłowska-Mamińska R, Szymona K, Król P. Evolving microbial communities in cellulose-fed microbial fuel cell. Energies, $2018 ; 11: 124$.

[36] Paitier A, Godain A, Lyon D, Haddour N. Microbial fuel cell anodic microbial population dynamics during MFC start-up. Biosensors and Bioelectronics 2017;92:357-63.

[37] Deka RK, Brautigam CA, Liu WZ, Tomchick DR, Norgard MV. Evidence for posttranslational protein flavinylation in the syphilis spirochete Treponema pallidum: structural and biochemical insights from the catalytic core of a periplasmic flavin-trafficking protein. MBio 2015; 6:e00519-15.

[38] Covington ED, Gelbmann CB, Kotloski NG, Gralnick ]A. An essential role for UshA in processing of extracellular flavin electron shuttles by Shewanella oneidensis. Mol Microbiol 2010;78:519-32.

[39] Keogh D, Lam LN, Doyle LE, Artur Matysik A, Pavagadhi S, Umashankar S, et al. Extracellular electron transfer powers Enterococcus faecalis biofilm metabolism. Mbio 2018;9:e00626-17.

[40] Schmoll M, Seibel C, Tisch D, Dorrer M, Kubicek CP. A novel class of peptide pheromone precursors in Ascomycetous fungi. Mol Microbiol 2010;77:1483-501.

[41] Light SH, Su L, Rivera-Lugo R, Cornejo ]A, Louie A, Lavarone AT, et al. A flavin-based extracellular electron transfer mechanism in diverse Gram-positive bacteria. Nature 2018;562:140-4.

[42] King KC, Brockhurst MA, Vasieva O, Paterson S, Betts A, Ford SA, et al. Rapid evolution of microbe-mediated protection against pathogens in a worm host. ISME ] 2016;10:1915-24.

[43] Firth N, Fink PD, Johnson L, Skurray RA. A lipoprotein signal peptide encoded by the staphylococcal conjugative plasmid pSK41 exhibits an activity resembling that of Enterococcus faecalis pheromone cAD1. J Bacteriol 1994;176:5871-3.

[44] Cetinkaya Y, Falk P, Mayhall CC. Vancomycin-resistant Enterococci. Clin Microbiol Rev 2000;13:686-707.

[45] Vasieva O, Goryanin I. Is there a function for a sex pheromone lipoprotein precursor? Preprint. Available from: https://www.researchgate.net/publication/328318964_Is_there_a_function_for_a_sex_pheromone_lipoprotein_precursor. Doi: 10.13140/RG.2.2.17245.87525/1. 